\title{
An analysis of confusion errors in naming letters under speed stress
}

\author{
KEITH E. STANOVICH \\ Oakland University, Rochester, Michigan 48063 \\ and \\ ROBERT G. PACHELLA and J. E. KEITH SMITH \\ University of Michigan, Ann Arbor, Michigan 48109
}

\begin{abstract}
Three subjects named six visually presented letters under two levels of speed stress. The obtained confusion matrices for each stress condition were fit by Luce's choice theory, which provided measures of stimulus similarity and response bias. Speed stress produced proportional increases in pairwise similarity measures but had no systematic effect on response biases. In Experiment 2, the same three subjects named the same letters under two levels of stimulus quality and a constant response-time deadline. As with speed stress, degrading the stimulus produced proportional increases in pairwise similarity measures but had no systematic effect on response biases. In Experiment 3, two of the subjects participating in Experiments 1 and 2 named the six letters under conditions where the probabilities of the letters were unequal. The letters toward which the subject had been most biased in Experiments 1 and 2 were assigned low probabilities, and the letters toward which he was least biased were assigned high probabilities. The result of this manipulation was to completely reverse the ordering of the response bias parameters of the Luce choice model. It is suggested that the present methodology provides a means of validating as psychological constructs the parameters of various mathematical models of stimulus recognition.
\end{abstract}

In recent years, there has been much interest in the theoretical problems associated with the use of reaction time as a dependent measure in information processing research. Attention has focused on the problem of how the interpretation of experimental effects might be affected by changes in a covariate measure-error rate. This problem has led to investigations of what is now called the speed-accuracy tradeoff, the reliable decline in accuracy as a function of increased speed of responding. Thus, such researchers as Edwards (1965) and Yellott (1971) have attempted to develop mathematical models that can account for the form of speed-accuracy tradeoff function. The aim has been to describe the quantitative function relating reaction time to an accuracy measure, usually expressed as some transformation of the number of errors. However, more can be gained from an analysis of the errors produced in an experiment than some simple summary

This research was supported by the Advanced Research Projects Agency, U. S. Department of Defense, and Monitored by the U. S. Air Force Office of Scientific Research under Contract (F44620-72-C-0019 with the University of Michigan's Human Performance Center. Portions of these data were presented at the Annual Meeting of the Midwestern Psychological Association, Chicago, May 1975. Requests for reprints should be sent to Robert G. Pachella, Human Performance Center, 330 Packard Road, Ann Arbor, Michigan 48104. statistic like percent correct. The limitations on analyzing reaction time error data have largely occurred only because of the extensive use of two response alternative tasks-the only tasks for which reaction time models make precise predictions. Therefore, the nature of specific errors in these tasks are not especially diagnostic. However, specific confusions among stimuli in multiple alternative identification tasks often provide information regarding the nature of processing in the task. For example, the similarity of the incorrect responses to the stimulus words in tachistoscopic recognition has implications for theoretical models of the word frequency effect (see Neisser, 1967, Chapter 5). Likewise, Rumelhart (Note 1) has developed and tested a multicomponent feature extraction model of perceptual recognition by examining its ability to predict the specific stimulus confusions occurring among a fixed set of tachistoscopically exposed letters. Thus it is often desirable to look at the specific kinds of confusions among stimuli that occur when errors are made.

Many investigators have applied well-known mathematical models of the recognition process to the data obtained from experiments where subjects must name tachistoscopically presented alphabetic characters. For example, Townsend (1971) tested the ability of three models (a pure guessing model, a sophisticated guessing model, and Luce's choice 
model) to predict the confusion matrix obtained from presentation of the entire uppercase English alphabet. The sophisticated guessing and choice models were fairly successful in predicting the obtained confusion matrix. This study and others have demonstrated the utility of qualitative analyses of stimulus confusions. With this result in mind, it becomes of interest to see whether such analyses can be meaningfully applied to the errors produced in experiments using reaction time as a dependent measure.

The question raised above speaks to an issue of fundamental importance to experimental psychology, namely, whether the data obtained from reaction-time and tachistoscopic tasks converge on the same processes. The method typically used to investigate this problem has been to see if variables affecting accuracy in a tachistoscopic recognition task will have similar effects on reaction time when the stimulus remains exposed and errors are minimal (Eriksen \& Hoffman, 1972). Another, perhaps more direct, method of examining the convergence of these two tasks would be to look at performance in a reaction-time task where subjects made an appreciable number of errors. This method would allow the investigator to compare the pattern of errors produced under these conditions with those produced in tachistoscopic tasks. The aim of the studies reported here was to develop a reliable reaction-time task that yielded a relatively large number of errors, making possible the application of various mathematical models of stimulus recognition.

The method adapted for use in the present series of experiments was the response-time deadline technique introduced by Fitts (1966) and extended by Pachella and Pew (1968). The technique involves defining a criterion time prior to each block of trials. The subject's task is to respond faster than the criterion time on each trial. He is given feedback subsequent to each trial indicating whether his response was fast or slow. In this manner, the experimenter achieves control over the subject's speed of responding. After a relatively small amount of practice with this procedure, subjects' mean response times are usually slightly faster than the criterion time. More important for present purposes, error rates increase reliably with decreases in mean response time (Pachella \& Pew, 1968). Thus, the procedure affords the experimenter relatively precise control over the percentage of errors produced in a given experiment. By merely changing the criterion time, an investigator can increase or decrease the number of errors made by a particular subject. Although previous work with this technique employed manual responses (Fitts, 1966; Pachella \& Pew, 1968), the experiments described below used a vocal naming response in order to minimize the effects of re- sponse processes and to permit presentation of a wide variety of stimuli.

One of the main purposes of this investigation was to use a mathematical model that has been successful in predicting stimulus confusions occurring under tachistoscopic conditions to analyze the confusion matrix obtained by using the response deadline reaction-time procedure described above. An ideal model for these purposes is Luce's choice model (Luce, 1959, 1963). The model is well known and has been applied to a variety of tasks, including tachistoscopic recognition, where it has been successful in predicting the confusion matrices obtained from presentation of alphabetic characters (Townsend, 1971; Rumelhart, Note 1). The model (Luce, 1963) postulates both pairwise similarity parameters and response bias parameters. The probability of a response $r_{j}$ to a given stimulus $s_{i}$ is assumed to be the strength of that response relative to the total strengths of all responses to the stimulus $s_{i}$. The strength of response $r_{j}$ is given by $\eta_{i j} \beta_{j}$, where $\eta_{i j}$ is a parameter reflecting the similarity between $s_{j}$ and $s_{j}$, and $\beta_{j}$ is the strength of the bias toward response $r_{j}$ independent of the stimulus presented. Under the assumptions of $\eta_{\mathrm{ij}}=\eta_{\mathrm{ji}}$ and $\eta_{\mathrm{ii}}=1$, the model has $\mathrm{N}(\mathrm{N}-1) / 2$ similarity parameters and $\mathrm{N}-1$ bias parameters (where $\mathrm{N}$ is the number of stimuli). Parameters can be estimated by maximum likelihood procedures that derive from the fact that the Luce choice model predicts the following property:

$$
n_{i j} n_{j k} n_{k i}=n_{j i} n_{i k} n_{k j} \text { for all } i, j, k,
$$

where $n_{i j}$ is the number of times that response $j$ is emitted to stimulus $i$. This property defines a quasisymmetric contingency table and maximum likelihood procedures exist for finding the best fitting $\mathrm{e}_{\mathrm{ij}}$, the expected frequencies (Bishop, Feinberg, \& Holland, 1975 , p. 289). Once the expected frequencies are solved for, maximum likelihood estimates of the parameters can be obtained by algebraic manipulation:

$$
\eta_{i j}=\sqrt{\frac{e_{i j} e_{j i}}{e_{i i} e_{j j}}} \text { and } \frac{\beta_{j}}{\beta_{i}}=\sqrt{\frac{e_{i j} e_{j j}}{e_{j i} e_{i i}}}
$$

It is particularly interesting to examine how the sensory and response parameters of the Luce model are affected by certain experimental mainpulations that are frequently used in reaction-time work. Thus, the general purpose of the present study was to see if a qualitative analysis of the types of errors produced under speed stress might yield additional information about the psychological mechanisms that underlie performance. The methodology in- 
volved experimentally controlling the subjects' speed of responding and then qualitatively analyzing the types of errors made under different speed constraints. Confusion matrices obtained under these conditions are analyzable via the same recognition models that have been useful in experiments employing tachistoscopic presentation and unspeeded responses. Experiment 1 investigated whether the errors produced in a vocal naming reaction-time experiment in which the subject is under speed stress could be accounted for in a reasonable way by a specific mathematical model of the recognition process. Experiments 2 and 3 extended the methodology to look at possible changes in stimulus confusions that might occur under a number of different experimental manipulations.

\section{EXPERIMENT 1}

\section{Method}

Apparatus. A PDP-1 computer controlled the presentation of stimuli and recorded response latencies by means of a voice key. The stimuli were the uppercase letters B, C, D, G, P, T presented on a Hewlett Packard 1311A display device. The letters were approximately $.5 \mathrm{~cm}$ high and $.25 \mathrm{~cm}$ wide. Subjects sat about $65 \mathrm{~cm}$ from the display screen. The experimenter sat behind the subject and recorded the responses on a microswitch keyboard.

Subjects. The subjects were three paid volunteers recruited on the University of Michigan campus.

Procedure. Each session consisted of four blocks of 200 trials. The sessions lasted approximately $1 \mathrm{~h}$ and were run on separate days. The first session was a practice day on which the vocal naming task and the response-time deadline procedure, to be used throughout the study, were introduced to the subject. Before every block, the subject was informed of an experimenterdefined response-time deadline which he was to beat on every trial. After each trial, the subject was given the feedback "fast" or "slow" in the lower left corner of the display device, depending upon whether he had emitted his response before or after the response-time deadline. The stimulus remained in view until the subject responded.

A different deadline, ranging from $340 \mathrm{msec}$ to $525 \mathrm{msec}$ was assigned on the first day of each of the four blocks. This procedure allowed the experimenter to choose two deadlines, one fast and one slow, to be used in the remainder of the experiment. These criterion times were chosen so that the slow deadline would result in $10 \%-15 \%$ errors and the fast deadline would yield $20 \%$ errors or more. This procedure resulted in deadlines of 410 and $360 \mathrm{msec}$ for subject D.Z., 410 and $370 \mathrm{msec}$ for subject R.S., and 370 and 340 msec for subject H.B.

The subjects then completed six sessions, using the individually chosen deadlines, each subject thus producing a total of $4,800 \mathrm{re}$ sponses. The six letters were presented with equal probability. Two consecutive blocks of each deadline condition were run on each day. The order of deadline conditions (two fast deadline blocks first or two slow deadline blocks first) alternated over days. The subjects were informed of the deadline to be used on each block. Three-minute rest periods were given between each block.

\section{Results and Discussion}

The deadline manipulation resulted in a differential speed and accuracy of performance. For example, subject D.Z. produced $35 \%$ errors and a mean reaction time of $342 \mathrm{msec}$ under the fast deadline, whereas the slow deadline resulted in $13 \%$ errors and a mean reaction time of $376 \mathrm{msec}$. The confusion matrix for each subject under each speed stress condition was analyzed separately. Optimum maximum-likelihood predictions for each matrix were produced on the basis of Luce's choice model. Figure 1 displays separately for each stimulus the predicted and observed proportion of responses given to each member of the stimulus set from the fast deadline condition of one of the subjects. Data from this particular subject will be presented throughout the remainder of the present paper as his performance was typical with respect to the pattern of observed and predicted errors across stimuli and he took part in all three experiments to be presented. The choice model fit his data no better or worse than those of either of the other subjects. For all three subjects, the model provides an excellent descriptive account of the data. The model accounted for better than $95 \%$ of the variance in both conditions of speed stress. The chi-square goodness of fit statistic for these fits ranged from 11.7 to 46.7 and averaged about 2.3 times the degrees of freedom (10), and, with 2,400 responses in each matrix, such a fit is more than reasonably acceptable. Thus, the model accounts for the data well, but not perfectly, i.e., the chi-square values indicate that the small residual variation is systematic. For subject D.Z., a major reason for the lack of fit was the inability of
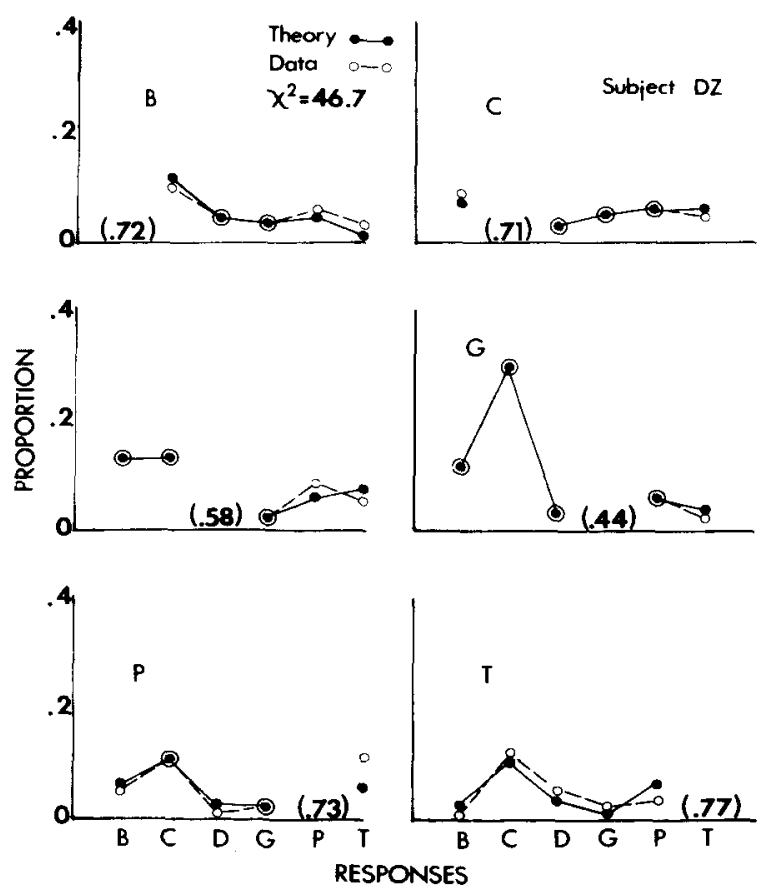

Figure 1. Subject D.Z.'s observed and predicted proportion of responses given to each member of the stimulus set from the fast deadline condition of Experiment 1. 
the model to accurately predict the proportion of $T$ responses. This pattern was apparent across all three subjects.

A more fine-grained analysis of the data is obtained by examining the effects of speed stress on specific parameters of the Luce choice model. The model has a response bias parameter for each of the stimuli and a similarity parameter for each pairwise combination of stimuli. The effect of speed stress on the similarity parameters is displayed in Figure 2, where the values of the similarity parameters in the fast deadline condition are plotted against the values of the similarity parameters in the slow deadline conditions. It is clear from Figure 2 that the model systematically reflects the increase in errors due to speed stress by roughly proportional increases in the similarity parameters in the fast deadline condition.

For each of the three subjects, the matrices from the two deadline conditions were refit using one set of bias parameters and two sets of similarity parameters. By comparing the fit of the Luce model under these conditions to that using two sets of bias parameters, one can test if a significantly better fit is obtained by estimating two sets of bias parameters. This analysis, carried out on the confusion matrices of subject D.Z., indicated that the goodness of fit was not significantly increased $\left[\chi^{2}(5)=10.4, p>.05\right]$ by estimating two sets of bias parameters. However, both subjects R.S. and H.B. displayed significant reductions in deviation from the model when an additional set of bias parameters was estimated from their data [R.S: $\chi^{2}(5)=20.5, \mathrm{p}<.01 ;$ H.B: $\chi^{2}(5)$ $=13.0, \mathrm{p}<.05]$. Although the preceding analyses

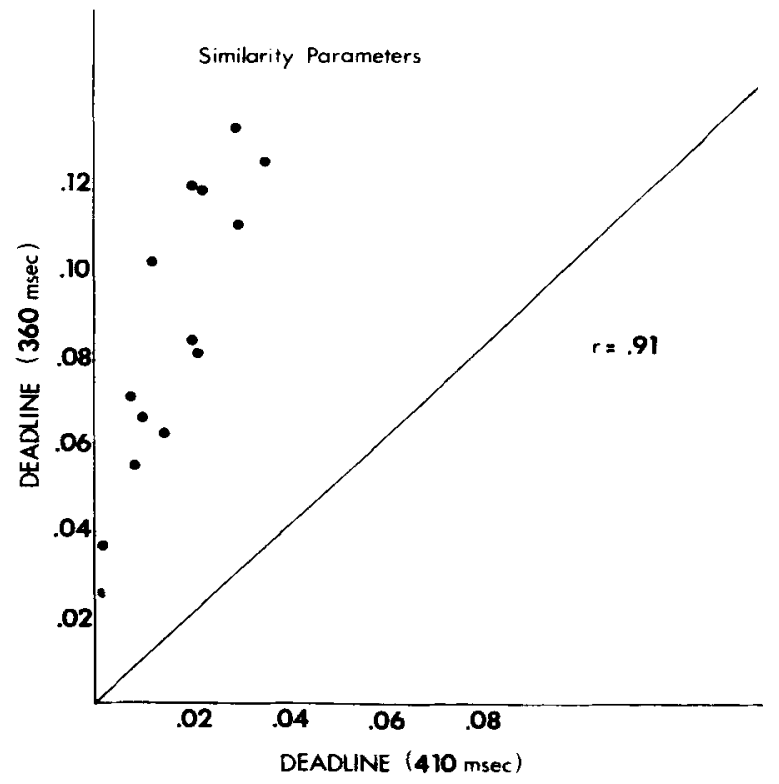

Figure 2. Values of the Luce similarity parameters in the fast deadline condition plotted against the same parameters from the slow deadline condition. Subject D.Z.

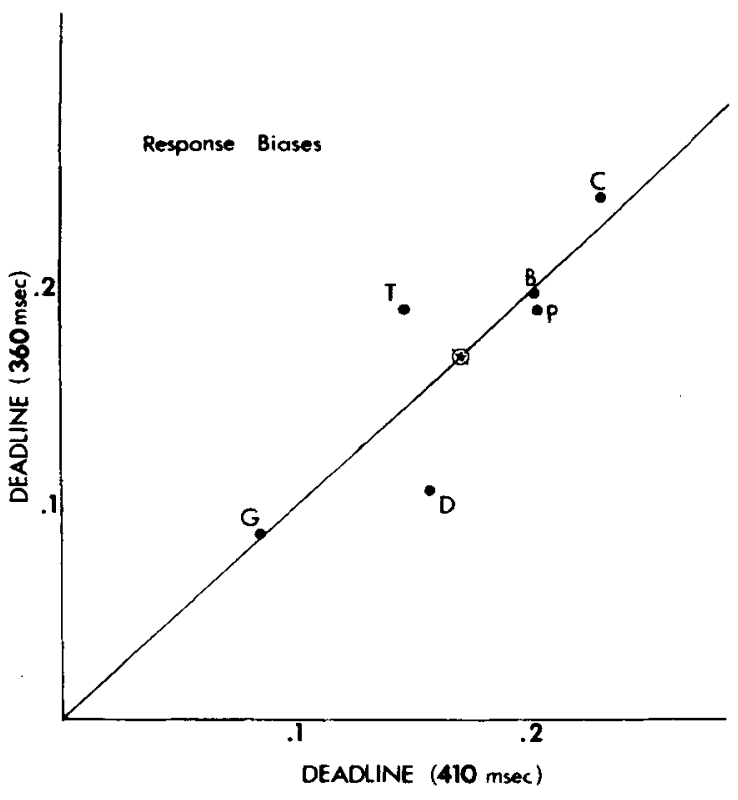

Figure 3. Values of the Luce response bias parameters in the fast deadline condition plotted against the same parameters from the slow deadline condition. Subject D.Z.

indicated that, for subjects R.S. and H.B., speed stress led to reliable changes in response biases, the differences in these parameters across experimental conditions did not seem to be in any way systematic. For example, lowering the response-time deadline did not result in a greater preponderance of the responses that were preferred under the more lenient deadline. Nor did the distribution of responses become more rectilinear with increased speed stress. Instead, the biases appear to differ reliably but unsystematically. Figure 3, which is a scatter plot of the response bias parameters in the two speed stress conditions for subject D.Z. is representative of all three subjects. It indicates that these parameters were not affected in any predictable way by changes in the response-time deadline. Thus, the increase in errors due to speed does not seem to be the result of simply a greater prevalence of the preferred responses. The confusions seem to be genuine and not the result of a simple fast-guess strategy.

As discussed earlier, the present methodology allows for investigation of several experimental factors that have been of interest in studies of stimulus recognition. In light of the results observed with the speed stress manipulation, Experiment 2 attempted to see if variation of stimulus quality would result in similar systematic effects on the parameters of Luce's choice model.

\section{EXPERIMENT 2}

\section{Method}

The procedure used in Experiment 2 was similar to that used in Experiment 1. The same three subjects who participated in 
Experiment 1 each completed six more sessions consisting of four blocks of 200 trials. The subjects named the same six letters under a constant deadline of $400 \mathrm{msec}$ for subjects D.Z. and R.S. and $350 \mathrm{msec}$ for subject H.B. On half of the trials, the stimulus was degraded by a method of contrast reduction. The reduction in contrast was achieved by placing a plastic sheet over the display screen, which reduced the stimulus intensity by $1.5 \log$ units. The manipulation of stimulus quality was blocked and the order of contrast conditions (two degraded blocks first or two normal contrast blocks first) alternated over days.

\section{Results and Discussion}

The method of manipulating stimulus quality under a constant deadline had the desired effect of producing differential error rates in the two experimental conditions. Subject D.Z. produced an error rate of $9 \%$ in the normal condition and $21 \%$ in the degraded condition. The confusion matrix for each subject under each speed stress condition was analyzed separately. Optimum maximum-likelihood predictions for each matrix were produced on the basis of Luce's choice model. Figure 4 presents the observed data and theoretical predictions from the degraded stimulus condition of subject D.Z. Here the model's fit is somewhat better than in the speed stress manipulation, the goodness of fit chi square not quite reaching statistical significance $\left[\chi^{2}(10)=\right.$ $16.1, \mathrm{p}>.05]$. Across all three subjects the ratio of the chi-square value to the degrees of freedom was approximately 2.0 .

The effect of stimulus quality on the similarity parameters of the Luce model is displayed in Figure 5. Here, as with the deadline manipulations, the effect of increasing errors due to degrading the stimulus was reflected in the model by proportional increases in the similarity parameters.

For each subject, the confusion matrices produced under the two conditions of stimulus quality were refit using one set of bias parameters and two sets of similarity parameters. The fit obtained under these conditions was compared with that resulting from estimation of a separate set of bias parameters for each matrix. For subjects D.Z. and H.B., significant reductions in deviation from the Luce model were obtained by estimating a second set of bias parameters [D.Z: $\chi^{2}(5)=11.5, \mathrm{p}<.05 ;$ H.B: $\left.\left.\chi^{2}(5)=18.7, \mathrm{p}<.01\right)\right]$. However, subject R.S. showed a nonsignificant reduction $\left[\chi^{2}(5)=10.4\right.$, $\mathrm{p}>.05]$. As in experiment 1 , the differences in response bias parameters across experimental conditions were in no way systematic. Figure 6 displays the effect of degrading the stimulus on the response bias parameters of subject D.Z. It indicates that there was no systematic effect on the response bias parameters as a result of degrading the stimulus. Thus, the increase in errors due to increasing speed stress or stimulus degradation can be accounted for by changes in the similarity parameters of the Luce choice model.
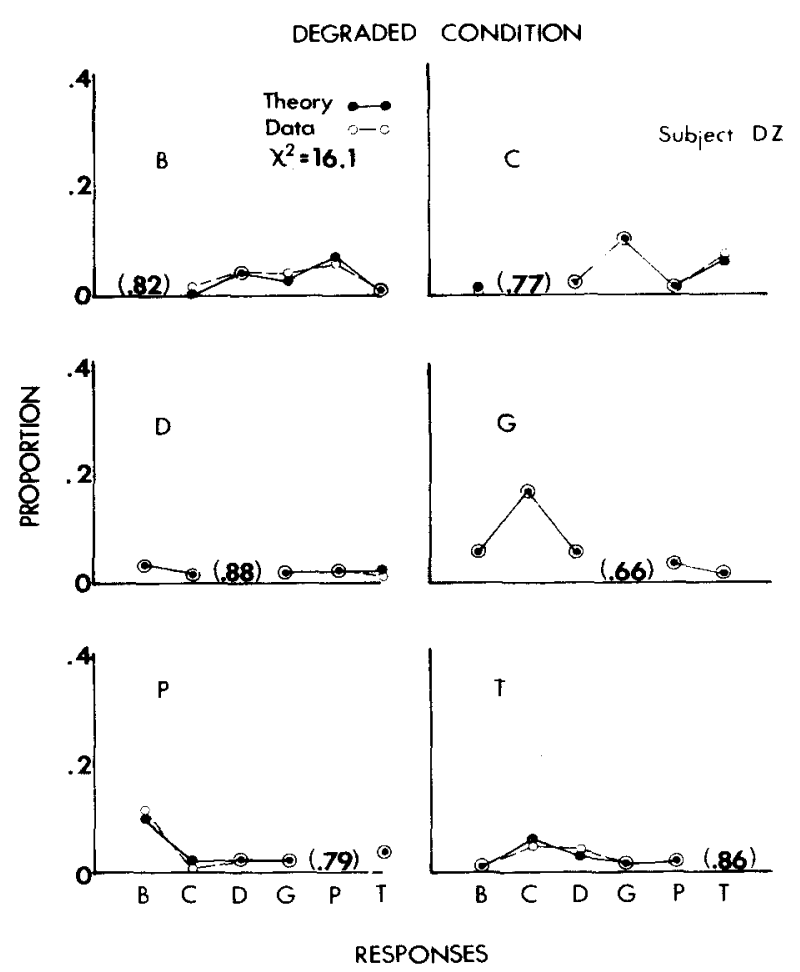

Figure 4. Observed and predicted proportion of responses given to each member of the stimulus set from the degraded stimulus condition of Experiment 2. Subject D.Z.

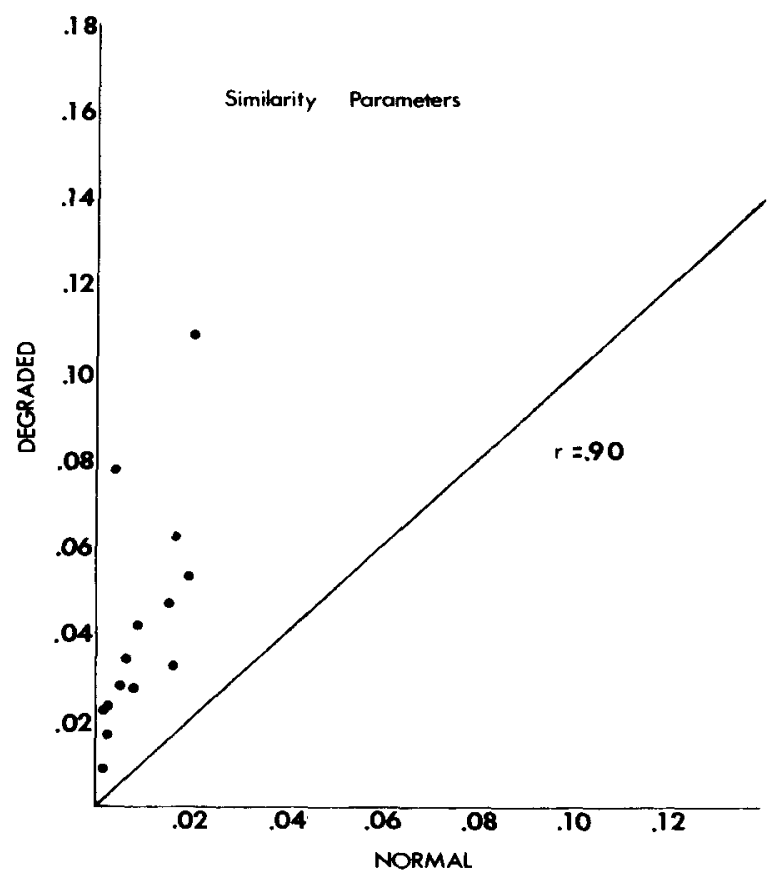

Figure 5. Values of the Luce similarity parameters in the degraded stimulus condition plotted against the same parameters from the normal stimulus condition. Subject D.Z. 


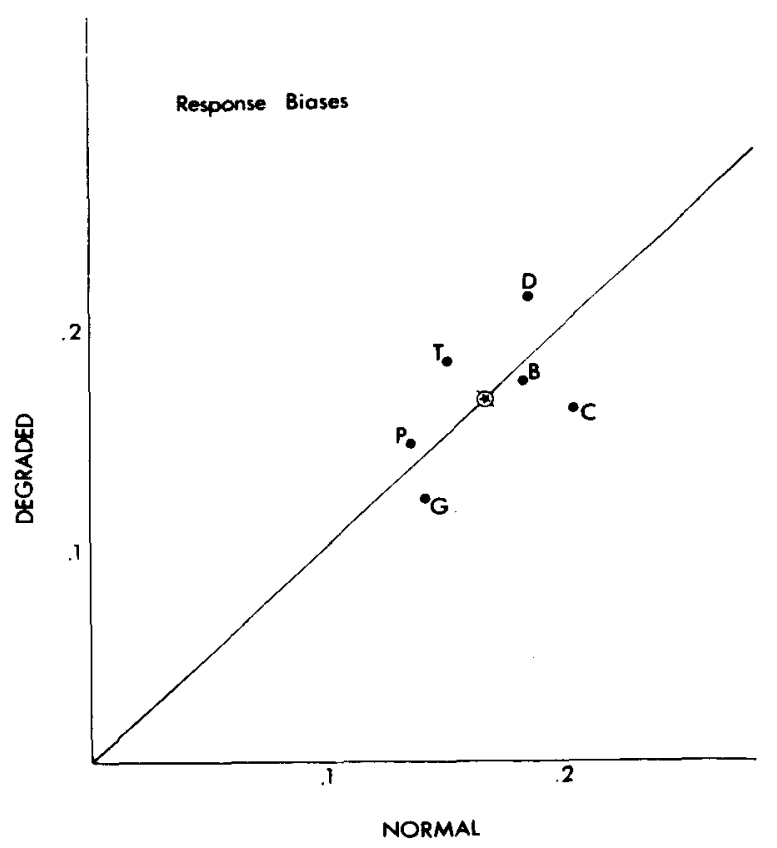

Figure 6. Values of the Luce response bias parameters in the degraded stimulus condition plotted against the same parameter from the normal stimulus condition. Subject D.Z.

\section{EXPERIMENT 3}

One final manipulation was run in an attempt to investigate a factor expected to systematically affect the subjects' response biases. This manipulation involved using unequal stimulus probabilities in the naming task.

\section{Method}

The procedure used in Experiment 3 was similar to that used in Experiments 1 and 2. Two of the subjects, D.Z. and R.S., who participated in Experiments 1 and 2, completed six additional 1 h h sessions employing a constant response-time deadline of 340 and $370 \mathrm{msec}$, respectively. For each subject, the two stimuli toward which he had been most biased during the previous experimental manipulations were each presented on only $5 \%$ of the trials. The two stimuli toward which he was least biased were each presented on $35 \%$ of the trials. The remaining two stimuli each appeared on $10 \%$ of the trials. The six sessions produced a total of 4,800 responses per subject.

\section{Results}

The confusion matrix obtained from each subject was analyzed separately. Optimum maximumlikelihood predictions for each matrix were produced on the basis of Luce's choice model. Figure 7 displays the data and the theoretical predictions from the performance of subject D.Z.

Figure 8 shows the effect of unbalancing the stimulus probabilities on the similarity parameters. The similarity parameter values of the $410-\mathrm{msec}$ deadline condition of Experiment 1, in which the stimuli had equal probabilities, are plotted against the parameters estimated from the six sessions of Experiment 3. The resultant scatterplot is very similar to that obtained in Experiment 1, where speed stress was manipulated. The parameters are linearly related and below the diagonal due to the faster deadline, and thus greater number of errors, in the unequal probability condition.

More interesting is the effect of unbalancing the stimulus probabilities on the response bias parameters of the Luce choice model. This effect is displayed in Figure 9. The method of placing highly preferred stimuli at low probabilities, and vice versa, had the effect of completely reversing the ordering of the response bias parameters of the Luce model. The increase in goodness of fit resulting from estimating a separate set of bias parameters for the unequal probability matrix, in addition to the biases from the 410-msec condition of Experiment 1, was highly significant for both subjects [D.Z: $(5)=643.9$, $\mathrm{p}<.001 ;$ R.S: $\chi(5)=74.7, \mathrm{p}<.001]$. Thus, although increasing speed stress or manipulating stimulus quality had no effect on response biases, changing the stimulus probabilities did have large and systematic effects.

\section{GENERAL DISCUSSION}

The experiments reported here establish several
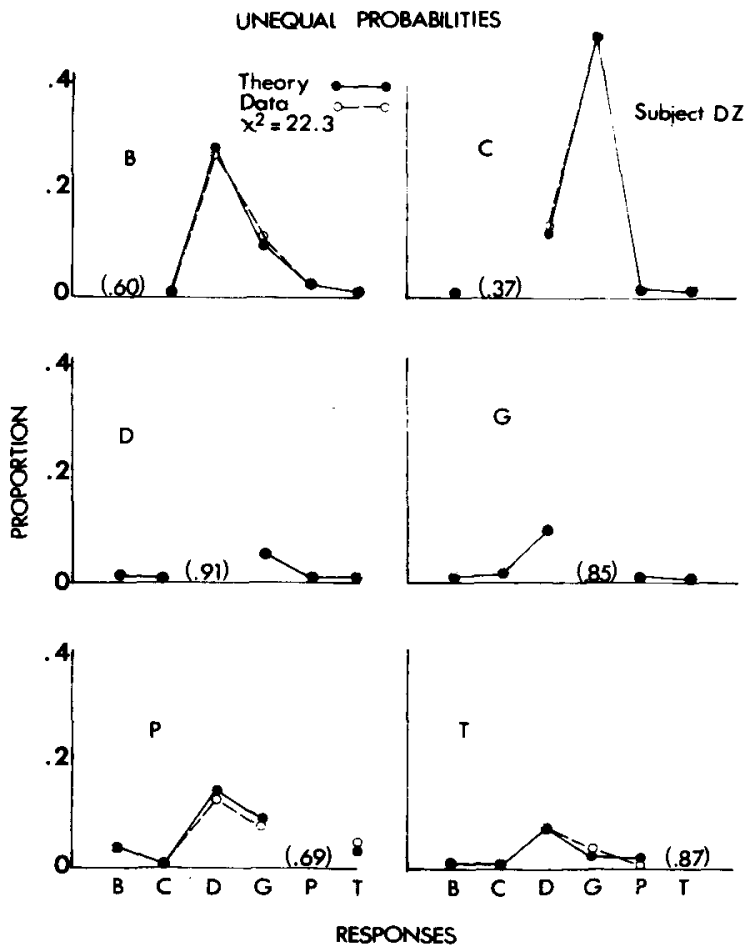

Figure 7. Observed and predicted proportion of responses given to each member of the stimulus set in Experiment 3. Subject D.Z. 


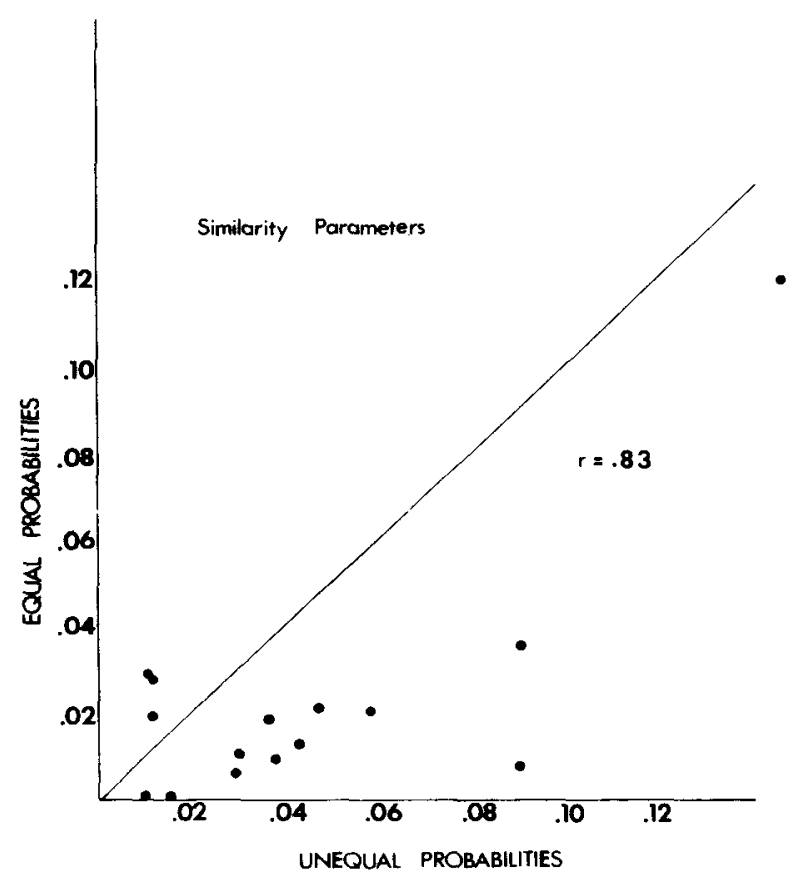

Figure 8. Values of the Luce similarity parameters in the 410msec deadline condition of Experiment 1 plotted against the same parameters from Experiment 3. Subject D.Z.

important facts about the response-time deadline experimental paradigm. First, subjects were able to perform the basic task. This result was not a trivial finding, since the application of the response-time deadline technique had not previously been attempted

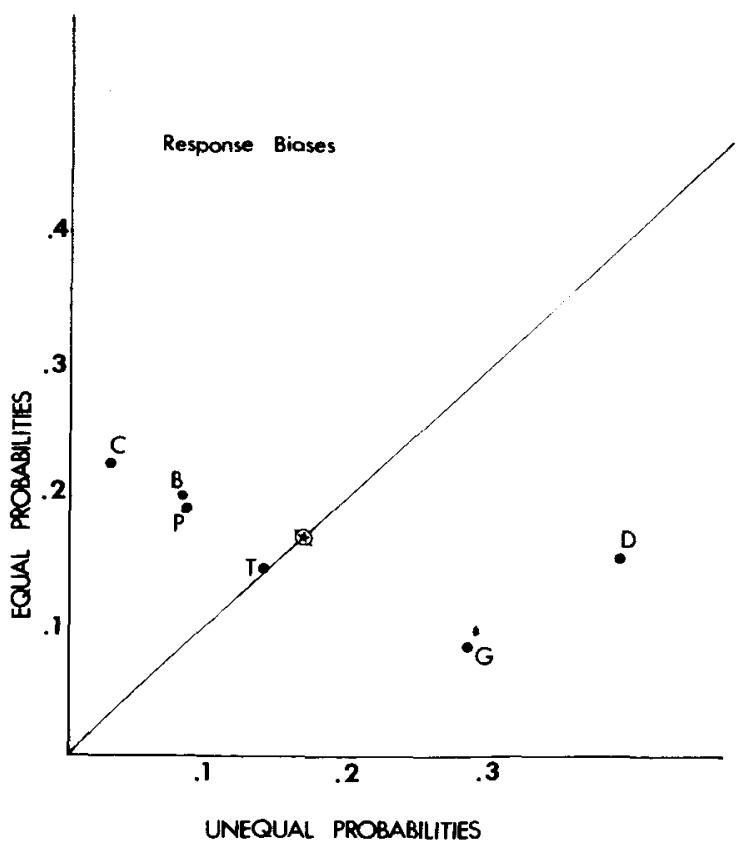

Figure 9. Values of the Luce response bias parameters in the 410-msec deadline condition of Experiment 1 plotted against the same parameters from Experiment 3, Subject D.Z. with verbal responding. Second, the subjects produced meaningful confusion matrices. That is, the confusions were clearly stimulus dependent and could not be fit with simple independence assumptions. This fact is also not trivial, since, on the basis of work by Yellott (1971) and Swensson (1972), subjects could have accommodated the speed demands by producing strings of essentially random guessing responses. This expectancy seemed particularly relevant since the earlier work (Swensson, 1972) had indicated that this strategy might tend to be associated with simple tasks using highly discriminable stimuli. Third, Luce's choice model provided a good description of the data. Finally, the parameters of the model reflected in a reasonable manner changes in experimental conditions.

One of the purposes of the investigation reported here was to provide a demonstration of a particular research strategy that has unique advantages over other methods. The paradigm is a variant of the more common procedure of looking at differences in reaction time across various experimental factors. Given the existence of the speed-accuracy tradeoff, the logic of the typical procedure can be reversed without modifying the interpretation of the resulting performance measure (Ollman, Note 2). Thus, a subject can be asked to produce responses of some constant duration across all conditions of an experiment. The resulting error rates can then be interpreted as indices of processing difficulty.

Under these circumstances, however, the error measures can actually yield much more information about the nature of the underlying mental operations than can the corresponding reaction-time measures. The reaction-time measures, when produced under errorless performance provide only an ordering of the level of difficulty of the experimental conditions. They produce no qualitative information about the relationships between various stimuli and responses. The error measures, on the other hand, not only produce the same ordering among the conditions, but additionally, they supply a descriptive profile of the specific confusions made by the subject. That is, from the distribution of the specific incorrect responses made to each stimulus, it is possible to reconstruct relationships between stimuli and responses from which detailed models of mental processing can be deduced. Many of these models (e.g., Luce's choice model) have had extensive theoretical and empirical development, and the application of these models to the errors produced in the context of reaction time experimentation can supply additional qualitative information that cannot be obtained from latency measures by themselves.

The present set of experiments demonstrates the utility of qualitative analyses of errors obtained in tasks requiring speeded responses. The analyses reported here are also a valuable first step toward 
construct validation of various mathematical models of stimulus recognition, a research strategy suggested by Nakatani (1973). Many models of this type assume two disjoint sets of experimental factors, one affecting stimulus parameters of the model and the other affecting bias parameters. Two sets of experimental factors applied to detection tasks have been demonstrated to have such effects on the parameters of signal detection theory (e.g., Green \& Swets, 1966). The present experiment has shown that disjoint sets of stimulus and bias factors exist for recognition tasks, under speeded response conditions.

\section{REFERENCES NOTES}

1. Rumelhart. D. E. A multicomponent theory of confusions among briefly exposed alphabetic characters. Center for Human Information Processing Technical Report No. 22, University of California, San Diego, 1971.

2. Ollman, R. Discovering how display variables affect decision performance. Paper presented at the Sixth International Symposium on Attention and Performance. Stockholm, June 1975.

\section{REFERENCES}

Bishop. Y. M. M., Feinberg, S. E.. \& Holland, P. Discrete multivariate anahisis: Theory and practice. Cambridge, Mass: MIT Press. 1975.

EDwaros. W. Optimal strategies for seeking information: Models for statistics, choice reaction time, and human information processing. Journal of Mathematical Psychology, 1965, 2, 312-329.

Eriksen. C. W., \& Hoffman, J. E. Some characteristics of selective attention in visual perception determined by vocal reaction time. Perception \& Psychophysics, 1972, 11, 169-171.

FITTs, P. M. Cognitive aspects of information processing: III. Set for speed versus accuracy. Journal of Experimental Psychology, 1966, 71, 849-857.

GreEN, D. M., \& Swets, J. Signal detection theory and psychophysics. New York: Wiley, 1966.

LUCE, R. D. Individual choice behavior. New York: Wiley, 1959.

LUCE, R. D. Detection and recognition. In R. D. Luce, R. R. Bush. \& E. Galanter (Eds.), Handbook of mathematical psychology (Vol. 1). New York: Wiley, 1963.

NaKaTANI. L. H. On the evaluation of models for the word-frequency effect. Psychological Review, 1973, 80. 195-202.

NeISSER. U. Cognitive psychology. New York: Appleton-CenturyCrofts, 1967.

Pachella, R. G. \& Pew, R. W. Speed-accuracy tradeoff in reaction time: Effect of discrete criterion times. Journal of Experimental Psychology, 1968, 76, 19-24.

Swensson. R. G. The elusive tradeoff: Speed versus accuracy in visual discrimination tasks. Perception \& Psychophysics, 1972, 12. 16-32.

TOWNSEND, J. T. Theoretical analysis of an alphabetic confusion matrix. Perception \& Psychophysics. 1971, 9, 40-50.

YELLOTT. J. I. Correction for fast guessing and the speed-accuracy trade-oft in choice reaction time. Joumal of Mathematical Psychology, 1971. 8. 159-199.

(Received for publication November 29, 1976; revision accepted March 24, 1977.) 